\title{
Contribution to a Symbiogenic Approach in Astrobiology
}

\author{
Francisco Carrapiço, Luísa Pereira, Telma Rodrigues \\ Universidade de Lisboa, Faculdade de Ciências, Departamento de Biologia Vegetal, Centro de Biologia Ambiental, \\ Bloco C2, Campo Grande, 1749-016 Lisboa, Portugal. email: fcarrapico@fc.ul.pt
}

\begin{abstract}
This article intends to continue our previous work on the symbiogenic approach to chemical and biological evolution. We believe that cooperative and synergistic processes were responsible, using terrestrial and extraterrestrial materials, for the creation of a large prebiotic pool, closely related to geochemical contexts, and intense interactions within. Probably, a series of synergistic and cooperative effects produced a wide source of creativity, and functional advantages that pushed the emergence of complex and functionally integrated biological systems, through the evolution of selforganization and auto-catalysis. It was only after this biochemical evolution of structures, which produced the informational capabilities necessary for self-replication, that the Darwinian mechanisms could arise. This way of perceiving the emergence of life follows the proposals regarding life's initial evolution in which the progenote proposed consisted in an open community of very diverse primitive cellular entities with intense symbiotic associations, antagonisms, and competition, and with a rapid and reticulate pattern of evolution. We believe this symbiogenic approach should be considered in the understanding of chemical and biological evolution. This discussion contributes to the development of astrobiological knowledge, since it gives other perspectives about life's appearance and development on Earth and elsewhere.
\end{abstract}

Keywords: astrobiology, symbiogenesis, origin of life

\section{INTRODUCTION}

Nearly a century after the Oparin-Haldane hypothesis, which established the scientific bases to understand the origin of life, this problem remains unsolved. Questions, like the ones highlighted by Fry ${ }^{1}$ and others, are recurrent in literature: When did life emerge on Earth? Which processes were involved in life's origin? What environmental and geochemical conditions did Earth display at that time? Did life exclusively emerge on Earth or was it brought from outer space? Which source(s), endogenous or exogenous, formed organic compounds? How to define the nature of the first living system(s)? Was the primitive atmosphere a reduced one? In which place(s) and environments did the emergence of life occur? What is the actual role of minerals? What was the predominant temperature at that time? How to explain the cyclic and integrated nature of the first living systems? Is life's emergence divine, naturalistic or by chance? Also, several difficulties have been pointed, namely, the smaller "time window" for life's emergence and the primitive soup dilute character ${ }^{1}$. Until now the scientific community, in general, has not yet decided which of the several proposals for the origin of life is more adequate.

The current origin of life studies must focus on the physical and chemical principles and possible naturalistic mechanisms guiding and constraining its emergence. We must simultaneously use bottom-up and top-down approaches, and rely on data from several scientific fields: geochemical and geophysical data concerning the primitive and present Earth and Solar System, fossil records, current Earth system dynamics, data from living forms interrelations and interdependencies, the comprehension of complex living systems characteristics, and how the three domains of life are related. We need to go forward and backward in time, in order to have a more coherent picture and also to have an open mind related to the understanding of the mechanisms that rule evolution in nature.

Currently, the main theoretical models on the origin of life follow the guidelines of investigation established by Oparin and Haldane, in the first two decades of the $20^{\text {th }}$ century. The Oparin-Haldane hypothesis and the majority of studies on the origins of life and prebiotic chemistry are divided between two approaches: the metabolism and the replication hypotheses. However, the dilemma between metabolism and replication shouldn't be the major focus of the issue. Also,

Instruments, Methods, and Missions for Astrobiology $X$

edited by Richard B. Hoover, Gilbert V. Levin, Alexei Y. Rozanov, Paul C. W. Davies

Proc. of SPIE Vol. 6694, 669406, (2007) · 0277-786X/07/\$18 - doi: 10.1117/12.734008

Proc. of SPIE Vol. 6694 669406-1 
until now, all research has been based on classic Darwinian principles, and guided by the notion of evolution as a strictly competitive and gradual process, without any cooperative or synergistic involvement ${ }^{2}$.

Although the Darwinian and neo-Darwinian perspectives have, so far, dominated the scene, it's becoming clear that they do not explain all the mechanisms and processes of evolution. Since we believe that different aspects are explained by metabolic and genetic models, we should now look for the common processes and principles that ruled the pre-biotic evolution, and question how evolution proceeded in such adverse environmental conditions. Thus, a new approach and vision are needed to solve this problem. In this task every contribution stemming from astrobiology is inestimable, since we believe life is a cosmic imperative $\mathrm{e}^{3,4}$.

\section{SEARCHING FOR A NEW IDEA ON EVOLUTION}

The science of complexity defies the selectionist hegemony, when argued that such a long-term trend towards increasing complexity suggests the presence of additional mechanism(s). Complexity should also be correlated with the functional synergies it produces (Bonner 1988, in Corning ${ }^{5}$ ). For Polanyi (1968, in Corning ${ }^{5}$ ), the causal dynamics involved in a complex living system construction are multileveled, that is, the "whole" properties are constrained and molded by the "parts", which are themselves also constrained and shaped by the inferior level properties of their constituents' materials, and by the laws of physics and chemistry.

One good example of a synergistic complex biological system is the symbiotic association Azolla-Anabaena. Azolla is a heterosporic floating pteridophyte presenting overlapping leaves, each with a floating and a submersed lobe. In the chlorophyllous dorsal lobe there is an ovoid cavity with a filamentous nitrogen-fixing cyanobacterium, usually referred to as Anabaena azollae, and several genera of bacteria. This leaf cavity behaves as both the physiological and dynamic interface unit of this symbiotic association where the main metabolic and energetic flows occur. In this sense, it can be considered as a natural microcosm, a special self-organized microsystem, with a well defined ecological structure. This symbiotic association can also be considered a successful co-evolved system, with the symbionts always present in the fern's life cycle, suggesting a phylogenetic parallel evolution of the relation partners, and can be considered as a typical example of a hereditary symbiosis. In this symbiotic association, complex ecological communities of permanent microorganisms co-operate along with the fern in the maintenance of the whole. New metabolic and organic capabilities are acquired and developed by the partners, which establish a new level of organization that goes beyond the individual capabilities of any individual partner.

The term synergy refers to the effects that parts (or individuals) cannot achieve alone, being interdependent effects, resulting in different "wholes". Synergy is like an "umbrella" for cooperative interactions, and can deal with mutualistics and parasitic combinatorial effects, emerging from the aggregation of multiple incremental contributions of individual parts or organisms ${ }^{5}$, and can in fact be observed in nature. One main characteristic of the living world is that entities in varied combinations are prodigious novelty generators. Synergies from several kinds had a significant creative role in evolution, being a marvellous source of evolutive novelty. The functional (selective) advantages associated with synergistic phenomena, while comprising a common functional principle, are also an important cause of the complex functionally organized systems in "progressive" temporal evolution ${ }^{5}$. In this context, symbiosis is a factor of evolutive change, giving rise rather suddenly to evolutionary novelty which, in our point of view, challenges several Darwinian and neo-Darwinian tenets, namely gradualism ${ }^{2}$. The synergies associated to symbiosis had a leading role in the complexification, which was already suggested by other authors $2,18,28,30,31,32,33,34,46$.

Also, as argued by Watson ${ }^{7}$, certain mechanisms of genetic variation (symbiosis and others), allowing the combination of pre-adapted genetic material, enables an evolutionary process that is algorithmically distinct from Darwinian gradualist framework. The differences between "compositional evolution" and "gradual evolution" derive from different underlying algorithmic principles. The author suggests expanding the framework of evolution by natural selection to include a greater range of algorithmic possibilities, and that compositional evolution should also be included in evolution by natural selection, thus breaking the equivalence between evolution and gradualism. Using evolutionary computation the author shows that compositional evolution foresees the evolving of certain kind of complex systems, specifically systems with modular interdependency that would be considered unevolvable under the gradualist framework. 
The majority of autocatalytic and self-organized phenomena are also open to natural selection, with functional synergy being the bridge connecting self-organization and natural selection ${ }^{5}$. It is important to note that, after symbiogenic processes have occurred, the newly evolved entity can again become the subject of selection ${ }^{8}$.

The Russian biologist Constantin Merezhkowsky, founder of Symbiogenesis theory, established the term "symbiogenesis," in 1909: " $\ldots$ the origin of organisms through the combination or association of two, or several, beings, who entered in symbiosis". The study of symbiosis in development, hereditary and evolution, established a new vision of the organism and evolution, markedly different from neo-Darwinian view, and was put in context by Symbiomics, a new multi-transdisciplinary evolutive science with contributions from biology, biochemistry, physiology, genetics, ecology, and evolution of biological systems and their interfaces. It places symbiogenesis in the evolution context of a post neo-Darwinian perspective ${ }^{10}$. This theory implies the central role of interactions, in which individuality (new entity) emerges through incorporation ${ }^{11}$. It involves horizontal mergers, which can be rapid, and, usually, discontinuous, creating permanent and irreversible changes, the ground for evolutive novelty. Something new arises through merging, being a unique or new metabolism or structure(s), which weren't present before symbiosis. Thus, a new entity reveals behaviour, previously unobserved in the separate components. The new entity can vertically evolve, but is always preceded by a horizontal merger between two or more entities ${ }^{8}$. Following these ideas several authors, not ignoring the Darwinian evolution mechanisms, have made some innovative approaches including symbiogenic evolutive principles namely, Barricelli (1957, in Fogel $\left.{ }^{12}\right)$ and others ${ }^{2,7,8,10,11,12,13,14,15,16,17,18, ~ 19, ~ 20, ~ 21, ~ 22 . ~}$

The symbiogenic concept allows an innovative approach for life origins and evolution, capable of being a fundamental rule in life's establishment and development on Earth and elsewhere. This implies a new paradigm for the comprehension of biological evolution. In the symbiotic relations, the central aspect is the creation of evolutive novelty (metabolic, anatomical and organismal) ${ }^{2}$. The theory of symbiogenesis states that the most probable cause for unexpected complex structures (dead or alive) lies in the association of lesser complicated parts ${ }^{23}$. The evolutionary changes can be explained by an integrated cooperation between organisms, in which symbiosis acts, not as an exception, but rather as the rule in nature ${ }^{2}$. According to Dubos \& $\operatorname{Kessler}^{20}$ symbiosis must be considered as a creative manifestation of a general biological phenomenon where "... the nutritional effects of symbiosis are not its most interesting manifestation. More remarkable is the fact that many symbiotic systems produce substances and structures that neither one of the two components produces when growing alone". In these symbiotic associations the association depends not only of the intrinsic symbionts properties, but also the internal and external system environmental conditions.

Symbiogenesis will be the evolutive mechanism and symbiosis the vehicle through which that mechanism unfolds ${ }^{24}$. This idea is based on the concept presented by Anton de Bary ${ }^{25}$ where symbiosis is defined as "the common life of different organisms". The synergies associated with symbiosis perform a leading role in complexity evolutions ${ }^{5}$. They are involved in first living organisms" emergence, in primitive prokaryotes mergers, and in the "progressive" temporal co-evolution of aquatic and terrestrial ecosystems. Symbiosis is a robust phenomenon, offering many opportunities for ecological niche occupation, otherwise unviable, and also having highly relevant survival purposes (defence, protection, nutrition, motility, reproduction, communication). We believe symbiosis overcomes the obstacle raised by Theodosius Dobzhansky, who stated, in 1962, that no theory of evolution leaving the mystery of adaptation unravelled should be acceptable (in Corning ${ }^{5}$ ).

At the turn to the $21^{\text {st }}$ century, several investigators advanced proposals for universalizing symbiogenesis as an evolutive mechanism. As far as we know, the first proposal was made by the astrophysicist Freeman Dyson ${ }^{19}$. More recently, Gontier $^{8}$ developed the universal symbiogenesis concept as a genuine alternative to universal selectionism, arguing that selectionist explanations highlight the models of ramification, speciation, and vertical transmission, considering vertical evolution as the rule, while horizontal evolution is the exception. The author considers that "Universal Symbiogenesis is the process where by new entities are introduced because of interactions between (different) previously independently existing entities. These interactions encompass horizontal mergings and the new entities that emerge because of this are called symbionts. The process is irreversible and discontinuous." ${ }^{.8}$. The universal symbiogenesis gives us a general analytical tool for the immense interactions between different entities, namely pre-biotic and biotic entities. According to these ideas, a symbiogenic approach to the prebiotic evolution and the origin of life should be seriously considered and developed as a new paradigm shift on evolution ${ }^{2}$. 


\section{SYMBIOGENESIS IN CHEMICAL AND BIOLOGICAL EVOLUTION}

Traditionally, chemical and biological evolution studies have been based in neo-Darwinian principles, which can lead us to a dead end situation for the global comprehension of life's emergence. Other approaches are necessary, like the symbiogenic approach, to enlarge the evolutive process knowledge. As proposed earlier ${ }^{24}$, a symbiogenic scenario could have been responsible for the development of pre-biotic processes towards primitive cells. We suggest a naturalistic explanation for the origin of life, through the evolution of complex and adaptative systems, with symbiogenic mechanisms that guided the chemical evolution through its physical and chemical natural constraints, besides the already postulated Darwinian ones. These ideas follow Dyson's hypothesis on the origin of life ${ }^{\mathbf{1 8}}$, based on symbiogenic concepts and invoking Lynn Margulis ${ }^{11}$ ideas. In his theory Dyson ${ }^{18}$ synthesises the metabolic and genetic visions of life's origin, supported by symbiosis, and proposes a prebiotic evolution accomplished by the independent formation of metabolic systems and self-replicating molecules that evolved together. In a specific moment in time, the metabolic systems became encapsulated in primitive amphiphilic vesicles. Later, some of the molecules were first synthesised as parasites and in time, they became symbionts, and underwent evolution together. Afterwards, the primitive selfreplicating molecules joined them, first as parasites and later as symbionts.

According to Shenhav et $a l^{\mathbf{2 6}}$, there is the possibility for a metabolic system to store and propagate information (compositional information) which they consider fundamental to overcome the dogma stating that any information mechanism transfer inferior to base-pair templating is implausible. They also question why we should insist on a dichotomous separation of metabolism and templating in the most primordial stages of life's emergence. Why not consider a mesobiotic entity (one with primordial molecular assemblies, and devoid of long catalytic biopolymers or long informational biopolymers -sequential information-, but endowed with both molecular and ensemble complexity) embodying primitive features from metabolism and replication? We believe such mesobiotic entities could be the result of a primitive symbiosis between primitive metabolism and primitive replication molecules, as initially proposed by Dyson $^{18}$.

When considering a double origin, involving metabolism and replication, accomplished in multiple origins on Earth, we can visualize metabolism conveying the compositional information, and replication the sequential information, and by symbioses between these two, a hybridization of their informational content could occur. This way, we believe in a double origin of metabolic systems, self-catalysed, and peptide based, that developed through successive symbioses between abiotic components and self-replicating and auto-catalysed molecules. In this sense, life did not emerge once, but in two steps, as metabolism and as genetic information, that ultimately combined in a symbiotic fashion. According to Eigen et al $\left(1979,1981\right.$, in Corning $\left.{ }^{5}\right)$, cooperative processes ruled the initial stages of evolution. The basic organic monomers required different component materials integration. Also, complex polymers were based in monomers fusions. The author also considered auto-catalysis and self-organization as preceding mutations and constitutes the most fundamental novel source in the formative stages of evolution. Kauffman ${ }^{27}$ considers self-organization as a pre-requisite for evolvability that creates the kinds of structures that can benefit from natural selection. This process prominently uses principles of self-organization and coherency, and in this way, self-organization and selection are two sources of order in nature, being natural partners.

Our ideas for a symbiogenic scenario also get support from other authors ${ }^{28}{ }^{29}$. Popa $^{28}$ argues that two major symbioses were involved in life's emergence. The first case of molecular symbiosis in life's evolution occurred through the association of a frontier (primitive membrane) with a reflexive activity, based on an association or mutual assistance between two distinct characteristics (entities). Metabolism emerges from the coupling between a self-assembly process and a reflexive activity that stabilizes it. Also, the reflexive activity was established by different synergisms and mutualisms (cooperation) through auto-catalytic cycles and hypercycles that several other authors propose as leading to the emergence of symbiosis. Eigen et al $\left(1979,1981\right.$, in Corning $\left.{ }^{5}\right)$, proposed the hypercycles as a way of explaining how organic substances with increasing complexity could overcome the "complexity catastrophe", displaying an example of synergy in evolution.

According to King ${ }^{30}$, auto-catalytic chemical systems can spontaneously arise, especially in an irradiated aqueous medium. Self-reproducing chemical particles, of any complexity, and in a proper environment, has self-regulating properties, allowing long-term survival. The environmental loss of materials, leading to continuous system decay, can be overcome through physical unions involving different kinds of self-reproducing particles. Such unions (symbioses) 
create an increasing and irreversible complexity, giving rise to chemical system evolution. The author suggests that evolution occurred through spontaneous successive symbioses. In this way, successive symbioses changed simple autocatalytic particles into primordial cells.

Also, Lee $e a^{31}$ argued that mutualistic symbiotic relations can operate at the molecular level, through hypercycles, a collection of two or more self-replicating species, interconnected through a cyclic catalytic network. The superimposition of cross-catalysis in auto-catalytic replication, will integrate hypercycle members in one system, which will reproduce through a second order (or higher) non-linear self-catalysis. The entire hypercycle population, as a whole, can compete more efficiently for resources, than a single member on its own. Besides, the positive mutation effects, of any member, are spread over the entire population. The hypercycles have been suggested as an important step in the transition from non-living chemistry to the world of living chemistry, and so, a huge number of hypercycles are expected to be embedded within living forms' complex networks.

Popa's ${ }^{28}$ second primeval symbiosis occurred between RNA molecules and abiotic peptides molecules (two different entities, a RNA like partner and a peptide like partner merged), triggering encription, the starting point for biological life. Only, after encription, did other properties arise: self-reproduction, total control over internal information, a genome creation and Darwinian evolution. According to Fernando ${ }^{32}$, if in a self-reproducing and auto-catalytic cycle, with a fundamental organization, two auto-catalytic replicating entities, previously independent, establish a physical union, still capable of auto-catalysis, and with a low decay rate, then symbiosis can still be viable in an environment with scarce resources, even if the symbiont's growth rate is slower than any of the component parts. The failing in establishing symbiosis can result in deficits, and will be a central problem for metabolism and template replication origins. Also, Bouchard $^{\mathbf{2 1}}$ argues that symbiosis is prevalent in nature, and a careful examination of the evolutionary strategies found in many obligate symbiotic associations validates a view of fitness that isn't understood in reproductive terms, but in ecological ones. According to the author, complex symbiotic systems can have an emergent fitness value described in terms of differential persistence of the community. In our opinion this vision of persistence is in favour of the importance of symbiogenic relations in withstanding extreme environmental conditions and the occupation of novel ecological niches.

For Woese ${ }^{29}$, two major evolutive transitions existed: the emergence of RNA World and the current cells world. These two transitions are associated with higher levels of organization, with new entities displaying new qualitative properties, and, in both, a complex network of sophisticated interactions (communication) at a distance, characterizing the medium of these new entities. In this way, translation (nucleic acids/peptide interactions) results from interaction fields that attain a certain complexity and specificity. Also, for Barricelli ${ }^{23}$, the software analogue in the living world is a self-replicating DNA molecule. Most of the software is parasitic (symbiotic), dependent upon metabolism, instead of freely replicating, strongly suggesting an analogy tolife.

The RNA World has been subjected to several criticisms, one being the difficulty of endogenous formation of nucleotides (although some think they could have been brought to Earth from elsewhere). Mathews ${ }^{33}$ tried to solve this issue through the establishment of a symbiotic process. As the author states "hydrogen cyanide polymers could be major components of the dark matter observed on many bodies in the outer solar system, including asteroids, comets, moons, rings and planets. Current studies on these ubiquitous compounds point to the presence of polyaminomalononitrile (I), a polyamidine structure built only from hydrogen cyanide. Cumulative reactions of HCN (or other reactive species) with the activated nitrile groups of I yield other polyamidines (II, with side chains R') which are converted stepwise by water to polypeptides (III, with side reactions R). Overall, this series of reactions constitutes a route for the direct synthesis of polypeptides from hydrogen cyanide and water without the intervening formation of $\alpha$-amino acids. Implications for prebiotic chemistry are profound. Primitive Earth may have been covered by HCN polymers as well as other organic compounds, either through bolide bombardment or by photochemical reactions in a reducing atmosphere. According to this model, membrane material - carboxylic acids, carbohydrates, and polypeptides - accumulated in lakes and oceans, while on land, polyamidines could have been the original dehydrating agents directing the synthesis of nucleosides and nucleotides from available sugars, phosphates and nitrogen bases. Most significant would have been the parallel synthesis of polypeptides and polynucleotides arising from the dehydrating action of polyamidines on nucleotides. Metabolic material - hardware - thus arose separately from genetic components - software - as proposed by Freeman Dyson. Subsequent interfacing, perhaps with the help of clays, then produced the first replicating protocells. On our dynamic planet this polypeptide-polynucleotide symbiosis mediated by polyamidines may have set the pattern for the 
evolution of protein-nucleic acid systems controlled by enzymes, the most characteristic mode of life nowadays. In 2003, the same author ${ }^{34}$ infers that, from hydrolysis of HCN polymers, there is some evidence for peptidic structures. Also, hydrolysis/pyrolysis gives rise to nitrogen heterocycles, including purines and pyrimidines found in nucleic acids today. According to the author some recent experimental results are consistent with this model of HCN polymer chemistry playing an essential role in the origin of life.

We agree with the idea that existing abiotic peptides assisted a symbiosis with nucleic acids, leading to translation apparatus emergence. In this way, encapsulated entities, analogous to modern cells, emerged based on a nucleic acid organization. The peptide/nucleic acids symbiosis allowed the emergence of a primitive translation apparatus, and, according to Woese $^{29}$ and Doolittle ${ }^{35}$, emerged as an imprecise and primitive replication of genomes, with a small number of genes, and with simple and primitive cellular designs of modular nature. This was permissive, poorly defined, and subjected to an unrestricted horizontal gene transfer (HGT) that would not cause the cellular organization to collapse. The components with certain form/function could be replaced by rough equivalents, the primitive representatives of some of the actual specific enzymes that could have only a specific class reaction. This is a period of ephemeral organismal genealogy. For Woese ${ }^{29}$, the world of primitive cells was like a vast ocean of cosmopolite genes, flowing in and out of evolving cellular entities, and others. We think these other entities could be particles like viruses, as already been proposed by Ryan ${ }^{13}$ and Villarreal ${ }^{36}$. This was an essentially communal evolution, with a reticulate and cooperatively collective pattern of evolution to which we add hybridization, symbiosis and synergisms. In fact, this provided the basis for a multilevel communication world.

This way of looking at the emergence of life goes beyond the proposals of Woese ${ }^{29}$ and Doolittle ${ }^{35}$ regarding the initial evolution of life, and supports the notion of multiple origins in straight correlation with the geochemical contexts, natural constraints, and environmental conditions. The kind of progenote proposed did not consist of one single type of organism, but of an open community of very diverse primitive cellular entities with intense symbiotic associations, antagonisms, and competition, and with a rapid and reticulate pattern of evolution. Other evidence for the widespread of these initial and universal synergistic and cooperative interactions is that, through symbiogenic mechanisms, microbial mats and stromatolites could form. These are the later of the first examples of a community ecological strategy based on cooperative and synergistic mechanisms to survive in the primitive Earth extreme environmental conditions.

\section{THE SIGNIFICANCE OF SYMBIOGENESIS IN ASTROBIOLOGY}

The symbiogenic approach in astrobiology context is based in several tenets. A currently debated question is the definition of life. According to the Astrobiology Primer ${ }^{37}$, a definition of life based only on extant living forms fails in not considering earlier life forms that preceded current cellular life. Also, NASA's ${ }^{38}$ definition of life only considers evolution through competitive processes, excluding symbiogenic ones, which does not reflect the reality in the natural world. The scientists' majority agrees that no single parameter can, by itself, define life, as life emerged from multiple mechanisms acting in concert. According to $\mathrm{Kolb}^{39}$, to define life as a phenomenon we must understand its origin and evolution from abiotic matter, through RNA World and LUCA. We agree, but we also believe that, in order to understand life as a phenomenon, we must consider some of its characteristics and properties.

According to our previous work and that of $\mathrm{Popa}^{28}$, we argue that life is a complex system, far from equilibrium, delimited from the exterior by a frontier, with vast information, with capabilities of self-assembly, self-catalysis, selforganization, self-reproduction and evolution. It has a self-sustained metabolism and homeostasis. It fights against dynamical equilibrium, and uses external sources of energy and nutrients. Its functioning is based on antagonisms, but also on synergistic and cooperative principles, as living beings establish associations and communicate with others. These are systems capable of competitive relations, and also of cooperation and varied synergisms, frequently when confronted with extreme environmental conditions ${ }^{40}$. We agree with Trainer $e t a l^{41}$, that life is an emergent global phenomenon on Earth, and that its emergence was a step by step process with rapid evolutive jumps within a small geological "time window", and subjected to physical-chemical constraints, intimately correlated with different geochemical contexts and environmental conditions, and supported by self-assembly, self-catalysis, liquid water and basic organic compounds. This follows from the ideas of Eldredge and Gould ${ }^{42}$. 
Facing the question "Is life a cosmic imperative?", we feel the answer is yes ${ }^{2,4}$. The symbiogenic approach to life's emergence contributes to the development of astrobiological knowledge, as it is able to give other perspectives on the study of life's appearance and development on Earth and elsewhere. "The Azolla-Anabaena-Bacteria System can be seen as a microcosm (small, partly isolated ecosystems...) inside of an organism and can also be considered as a good example of a living model for biological and environmental studies. The nature and the complexity of this system can also be seen as a reference for the design of new environmental engineered symbiotic systems that include man as a prelude to life in space. The fern enable high performance for some specific metabolic reactions of the symbionts, namely nitrogen fixation, ammonium and hydrogen production by the immobilized cyanobiont. Further, the use of Azolla as a wastewater biofilter and in biologically based life support systems (BLSS) incorporated in bioregenerative space devices are now in progress in several laboratories. The first clear indication that Azolla-Anabaena system could be used with success in a BLSS was shown in the 1980 seven-days space mission of the joint Soviet-Vietnamese cosmonauts crew Salyut 6 - Soyuz 37. Azolla pinnata was exposed to the weightlessness of space and the results were quite positive. The conditions of microgravity did not affect the main biological characteristics of either the fern or the cyanobacteria, as well the subsequent generations of Azolla on Earth. All these data strongly suggest that this symbiotic association should be used in a BLSS for space exploration involving men"433. Also, "Lichens seem to be well pre-adapted to deal with the extreme conditions of space including vacuum, abrupt temperature changes, and intense UV and cosmic radiation. Given their high level of resistance, lichen symbiotic organisms may be model organisms for interplanetary transfer"44. The experiments performed with lichens by the authors at Biopan-5 Facility, after exposure to space, suggest that, so far, these lichenized fungal and algal cells are able to survive the conditions of space ${ }^{44}$. The importance of symbiosis for survival is still currently visible. One example is that of the submarine hydrothermal vent communities discovered in 1977. This diverse biocenose supported by symbiotic chemolitotrophic microorganisms, live in total darkness. In these extreme environmental conditions, the established symbiotic relations between organisms from different domains of life allow these organisms to thrive in this unthinkable environment. These examples restate the symbiosis' importance when living forms are confronted with rapid and radical environmental changes. It also leads us to consider whether symbiogenic principles, namely the establishment of symbiosis, constituted a general rule under primitive Earth conditions.

Our planet can be considered as an open ecological system, with natural subsystems (lithosphere, atmosphere and hydrosphere), also open and interdependent, with constant exchanges of matter and energy between them and with the universe, that evolve and cooperate, keeping Earth in a dynamical equilibrium. We must consider that this planet's dynamic was also present on primitive Earth. The fourth natural system is the biosphere, and it crosses all the others, being supported by their interactions. Looking at Earth nowadays, we are confronted with an immense diversity (biological and environmental), with huge facets, interactions, synergistic phenomena, and antagonisms, under a variety of environmental conditions, some of which are considered extremes. Just as in the modern world, on primitive Earth there certainly occurred diverse chemical transformations and interrelations between the Earth natural systems, with important phase transitions at their interfaces, which resulted in their current interdependencies. Also, the three natural Earth subsystems, synergistically with extraterrestrial sources, provided an abundant input of organic compounds for prebiotic evolution. We should assume a diverse reality in primitive Earth, with different environmental characteristics, and multiple ecological niches, under different organic compounds concentrations.

We must assume the emergence of life on Earth not as an isolated phenomena in one specific place, but as a naturalistic one that occured in several places and times, and was guided by the universal principles mentioned above to overcome the natural constraints, favouring an increasing and irreversible diversity and complexity, in balance with the geochemical context and the environmental conditions. According to Lehto ${ }^{45}$, we can conceive of the locations for the different emergence steps toward life as being locally or temporally separated. In this way, several of the different scenarios proposed by different theories can be integrated in a convergent approach to the problem.

Several authors have already suggested some possible locations for the emergence of the first living systems, namely: volcanic fissures, mineral superficies, deep sediments, shallow lagoons, hydrothermal vents and springs, intertidal zones, ocean surface, aerosols surfaces, in the subsurface and in comets and asteroids. The existence of several changing habitats in the prebiotic Earth, with semi-permeable geographic frontiers, created environmental challenges that resulted in increasing complex and diverse entities. We believe that cooperative, synergistic and communicational processes were responsible, using terrestrial and extraterrestrial materials, for the creation of a large prebiotic pool, closely related to geochemical contexts and environmental conditions, and with intense interactions. 
According to Woese $^{29}$, cellular evolution did begin in a highly multiplex fashion, starting from independent ancestral communities. In this context, we envision a life emergence accomplished by multiple origins, in different times and environments, displaying a variety of selective contexts, which optimized symbiogenic processes in the promotion of creative novelty.

\section{FINAL REMARKS}

We consider that competition and cooperation can co-exist in the same scenario of evolution, and probably take place in discontinuous bursts of activity, depending on the internal and external conditions that drives evolution. It means that the same population can evolve using competitive and/or cooperative processes during the time and space of a hypercycle evolutive scenario. Thus, a series of synergistic and cooperative effects produced a wide source of creativity and functional advantages that pushed the emergence of complex and functionally integrated biological systems through the evolution of self-organization, self-catalysis and higher complexity. It was only after this biochemical evolution of structures, which gained informational capabilities necessary for self-replication, that Darwinian competition arose. Thus, the synergies associated to symbiosis had a leading role in the evolutionary complexification (fig. 1).

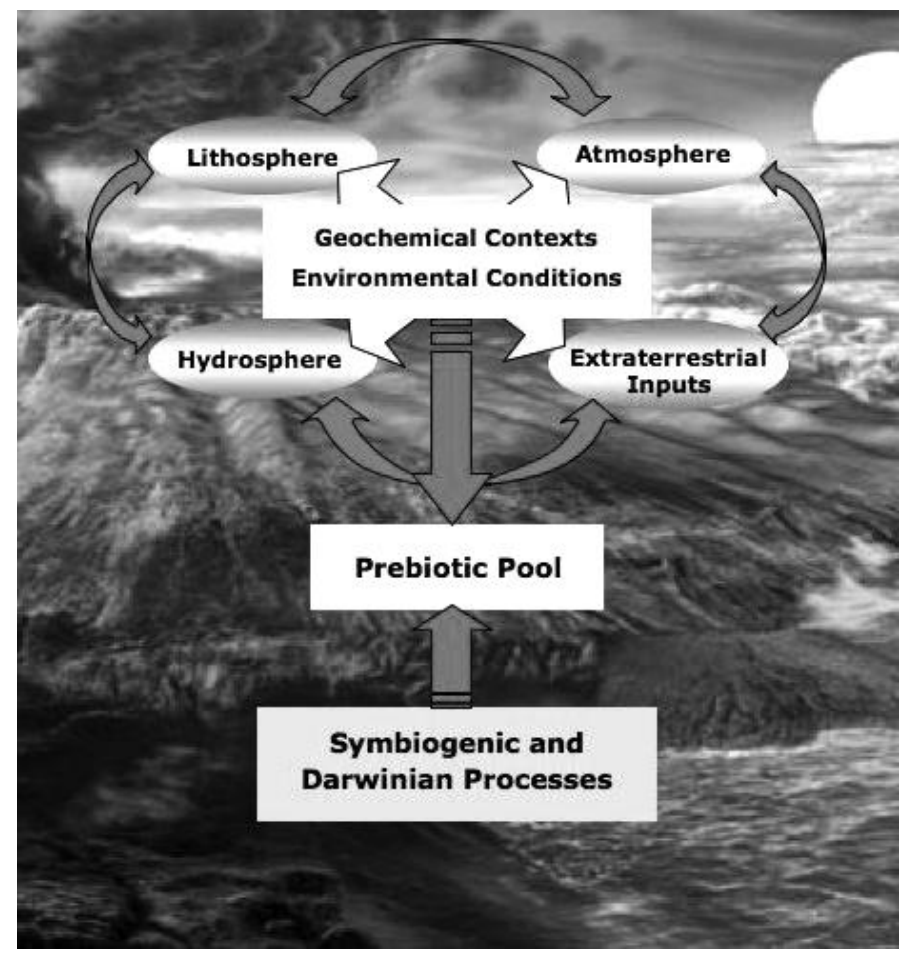

Fig. 1 - A scheme of the possible interactions in the prebiotic environment of the primitive Earth.

According to Corning 5 , varied synergistic effects were a primary cause for the observed tendency towards multifunctional, multileveled, hierarchically organized and increasingly complex systems. The complexification in evolution implies the production of new forms (entities) and more powerful synergies. In this context, symbiogenesis creates new important selection units (symbiomes) ${ }^{\mathbf{1 0}}$ arising through the integration of varied parts and progressive differentiation of the whole, providing an evolutionary advantage that goes beyond the traditional neo-Darwinian units of selection.

According to Fry ${ }^{1}$ the "arch and scaffold" model constitutes a metaphor to analyse the origin of life theories. Following this idea we suggest that the synergistic, cooperative and symbiogenic interactions between the prebiotic entities 
contributed to the establishment of the physical and chemical scaffolds the author talks about. In our perspective, those scaffolds or supports, together with the physical and chemical pre-biotic constraints, the geochemical compatibilities, the environmental conditions, coupled with Darwinian evolutive mechanisms resulted in the current domains of life. These ideas lead us to propose a new theory for the comprehension of life's emergence and its initial evolution. We call it the Symbio-Darwinian Theory.

We also believe that the existing models proposed for life's origin and evolution are different but complementary for the same reality, applied to the primitive Earth. Thus, a more cooperative and communicational work is necessary to help reveal the so far hidden answer(s), and for the search of life elsewhere. Astrobiology, being a multi-transdisciplinary science, has the conditions to be the primary actor leading this enterprise and to implement this paradigm shift.

\section{ACKNOWLEDGEMENTS}

The authors are grateful and express their sincere thanks to Dr. Gilbert Levin and Dr. Richard Hoover for the support and encouragement related to this work. A particular thanks to Helena Carrapiço for the English revision of the manuscript.

\section{BIBLIOGRAPHY}

1. I. Fry, The Emergence of Life on Earth. A historical and scientific overview, Free Association Books, London, 2000.

2. F. Carrapiço, "The Origins of Life and the Mechanisms of Biological Evolution", Proceedings of SPIE 6309, 63090O1 - 63090O-5, (2006b).

3. G. V. Levin, "Mars life - how Darwinian pressures might have shaped its form and function", Proceedings of SPIE 5906, 59060D-1 - 59060D-10 (2005).

4. R. B. Hoover, "Mineralized remains of morphotypes of filementaous cyanobacteria in carbonaceous meteorites", , Proceedings of SPIE 5906, 59060J-1 - 59060J-17 (2005).

5. P. A. Corning, "Synergy and Self-organization in the Evolution of Complex Systems", Systems Research 12 (2): 89121 (1995).

6. F. Carrapiço, "Is the Azolla-Anabaena Symbiosis a Co-evolution Case?" General Botany: Traditions and Perspectives. Materials of the International Conference, dedicated to $200^{\text {th }}$ anniversary of the Kazan Botanical School. Edited by Andrew Sitnykov. Part I, 193:195. Published by the Kazan University, Russia. Kazan (2006a).

7. R. A. Watson, "Compositional Evolution and Symbiosis", Abstract of ISHPSSB, 2007 Meeting, University of Exeter, pp. 183-184.

8. N. Gontier, "Universal Symbiogenesis: An alternative to universal selectionist accounts of evolution" Symbiosis, 44, (in press), (2007).

9. J. Sapp, F. Carrapiço, M. Zolotonosov, "Symbiogenesis: The Hidden Face of Constantin Merezhkowsky", Hist. Phil. Life Sci., 24, 413-440, 2002.

10. J. Sapp, Genesis: The Evolution of Biology, Oxford University Press, New York, 2003.

11. L. Margulis, The Symbiotic Planet, a New look at Evolution, Phoenix, Orion Books, London, 1999.

12. D. B. Fogel, "Nils Barricelli - Artificial Life, Coevolution, Self-adaptation", Historic Perspective, IEEE Computational Intelligence Magazine, February (2006).

13. F. P. Ryan, "Genomic Creativity and Natural Selection: a modern synthesis", Biological Journal of the Linnean Society, $88: 655-672,(2006)$.

14. M. J. Chapman and L. Margulis, "Morphogenesis by Symbiogenesis", International Microbiology 1: 319-326, (1998).

15. T. Taylor, "From Artificial Evolution to Artificial Life", PhD Thesis, Division of Informatics, University of Edinburg, 1999. http://www.tim-taylor.com/papers/thesis/html/ (25.10.06).

16. P. L. Antonelli, L. Bevilacqua, S. F. Rutz, "Theories and Models in Symbiogenesis". Nonlinear Analysis: Real World Application, 4, 743-753, (2003).

17. M. J. Roossinck, "Symbiosis versus Competition in Plant Virus Evolution". Nature Reviews, Microbiology, vol. 3, 917-924, December (2005).

18. F. Dyson, Origins of Life, Cambridge University Press, Cambridge, 1985.

Proc. of SPIE Vol. 6694 669406-9 
19. F. Dyson, Evolution, Society, Science and the Universe, Darwin College, Cambridge University Press, Cambridge, 1998.

20. R. Dubos and A. Kessler, "Integrative and Disintegrative Factors in Symbiotic Associations". Proceeding of the Thirteen Symposium of the Society for General Microbiology, Nutman, P. S. \& Mosse, B. (eds), p. 1-11, London, 1963.

21. F. Bouchard, "What is a symbiotic superorganism and how do you measure its fitness?" Abstract of ISHPSSB, 2007 Meeting, University of Exeter, p. 45.

22. J. Sapp, "The Dynamics of Symbiosis: an historical overview". Can. J. Bot., 82, 1046-1056, (2004).

23. G. B. Dyson, Darwin Among the Machines. The Evolution of Global Intelligency (Chapter 7), p. 111-130. Perseus Books (Ed), Cambridge, Massachusetts, 340p, 1997.

24. F. Carrapiço and T. Rodrigues, "Symbiogenesis and The early Evolution of Life". Proceedings of SPIE 5906, 59060R-1 - 5900R-4, (2005).

25. A. de Bary, "Ueber Symbiose", Tageblatt 51 Versamml. Deutscher Naturforscher u. Aerzte, Cassel, 121-126, 1878.

26. B. Shenhav, D. Segré, D. Lancet, "Mesobiotic Emergence: molecular and ensemble complexity in early evolution", Advances in Complex Systems", 6, (1), 15-25, World Scientific Publishing Company, (2003).

27. S. Kauffman, At Home in the Universe. The Search for Laws of Self-Organization and Complexity, p.321, Viking, 1995.

28. R. Popa, Between Necessity and Probability: searching for the definition and origin of life, Springer, Physics and Astronomy, Advances in Astrobiology and Biogeophysics, Springer-Verlag Berlin Heidelberg, 2004.

29. C. R. Woese, "A new Biology for a New Century", Microbiology and Molecular Biology Reviews, American Society for Microbiology, p. 173-186, June, (2004).

30. G. A. M. King, "Symbiosis and the Origin of Life". Origins of Life, 8, 39-53, (1977).

31. D. H. Lee, K. Severin, Y. Yokobayashi, M. R Ghadiri, "Emergence of Symbiosis in peptide self-replication through a Hypercyclic Network". Nature D. 11; 390 (6660): 591-4, (1997).

32. C. Fernando, "The Good Symbiont", M Caparrere et al (eds), ECAL2005, LNAI, 3630, pp. 695-704, Springer-verlag Berlin Heidelberg, 2005.

33. C. N. Matthews, "The HCN World: Establishing Protein-Nucleic Acid Life", The Alexander Ivanovich Oparin 100 ${ }^{\text {th }}$ Anniversary Conference, Miramare, Trieste, (1994).

34. C. N. Matthews, and R. D. Minard, "Prebiotic and Extraterrestrial Chemistry of Hydrogen Cyanide Polymers". Workshop on Cometary Dust in Astrophysics, p. 6012, (2003).

35. W. F. Doolittle, "Uprooting the Tree of Life". Scientific American, February, 90-95, (2000).

36. L. P. Villarreal, Viruses and the Evolution of Life, ASM Press, 2005.

37. "The Astrobiology Primer: An Outline of General Knowledge" - version 1, Education Paper, Mary Ann Liebert, Inc, 2006.

38. http://nai.arc.nasa.gov/astrobio/astrobio detail.cfm?ID=1399 (2007)

39. V. M. Kolb, "On the applicability of the Aristotelian principles to the definition of life", International Journal of Astrobiology, 6 (1): 51-57, Cambridge University Press, Cambridge, (2007).

40. F. Carrapiço, "A Origem da vida e a sua evolução. Uma questão central no âmbito da exobiologia", Anomalia 5, 2532, (2001).

41. M. G. Trainer, A. A. Pavlov, H. L. DeWitt, J. L. Jimenez, C. P. Mckay, O. B. Toon, M. A. Tolbert, "Organic haze on Titan an the early Earth", PNAS, November 28, Vol. 103, no 48, 18035-18042, (2006).

42. N. Eldredge and S. J. Gould, "Punctuated Equilibria: An Alternative to Phyletic Gradualism", Models in Paleontology, T. J. M. Schopf (ed), San Francisco: Freeman, Cooper, 82-115, (1972).

43. F. Carrapiço, "The Azolla-Anabaena-Bacteria System as a Natural Microcosms", Proceedings of SPIE, 4495: 261$265,(2002)$.

44. C. Ascaso, A. do los Rios, J. Wierzchos. C. Risueño, G. Horneck, R. de la Torre, L. G. Sancho, "Ultrastructural features of Rhizocarpon geographicum symbionts after exposure to space at the Biopan-5 facility", Abstracts from $6^{\text {th }}$ European Workshop on Astrobiology, Ecole Normale Supérieure de Lyon, France, October (2006).

45. K. Lehto, "From Molecular Evolution to Cellular Life" (Chapter 4, p. 85-120), Complete Course in Astrobiology, Physics Text Book, G. Horneck and P. Rettberg (eds), p 413, Wiley-vch, Verlag Gmbh \& co.KGaA, (2007).

46. S. A. Frank, "The Origin of Synergistic Symbiosis", J. Theor. Biol. 176, 403-410, (1995). 